\title{
Concise Commentary: Is Nrf2 a Master Regulator of Intestinal Fibrosis?
}

\author{
Giovanni Latella ${ }^{1}$
}

Published online: 19 December 2017

(C) Springer Science+Business Media, LLC, part of Springer Nature 2017

Intestinal fibrosis becomes clinically evident in approximately $40 \%$ of patients with Crohn's disease (CD) and in 5\% of those with ulcerative colitis (UC). Fibrosis, a consequence of local chronic inflammation, is characterized by excessive deposition of extracellular matrix (ECM) protein.

Although the transforming growth factor $\beta 1$ (TGF- $\beta 1$ )/ small mothers against decapentaplegic (Smad) pathway is the main driving force for fibrosis, several pro- and antifibrogenic molecules may also directly interact with this pathway $[1,2]$. Emerging evidence indicates that reactive oxygen species/reactive nitrogen species (ROS/RNS) and nuclear factor erythroid-2-related factor 2 (Nrf2) are connected with TGF during fibrosis development in numerous organs, including the intestine [3-6]. Nrf2 is an ubiquitous master transcription factor that upregulates the expression of antioxidant enzymes and cytoprotective proteins by antioxidant response elements (ARE). An impaired Nrf2 response has been described in several fibrotic diseases involving the liver, lung, kidney, heart, and arteries.

The Nrf2 pathway has the ability to alleviate acute colitis, chronic colitis, and associated colorectal cancer (CRC) in mouse models, but whether it can protect against colitisassociated fibrosis remains to be clarified. In this issue of Digestive Diseases and Sciences, Guan et al. [7] evaluated the effects of tert-butylhydroquinone (tBHQ) on colorectal fibrosis in mice with 2,4,6-trinitrobenzene sulfonic acid (TNBS)-induced chronic colitis. The Nrf2 inducer tBHQ, a widely used antioxidant food additive that induces the expression of Nrf2 and Nrf2-related genes in several organs including mouse intestines, significantly attenuated colorectal inflammation and fibrosis, as shown by reduced expression of myeloperoxidase activity, tumor necrosis factor $\alpha$ (TNF- $\alpha$ ), interleukin- $1 \beta$ (IL- $1 \beta$ ), collagen, and $\alpha$-smooth muscle actin ( $\alpha$-SMA, a marker of activated myofibroblasts).

Giovanni Latella

giolatel@tin.it

1 Gastroenterology Unit, Department of Life, Health and Environmental Sciences, University of L'Aquila, Piazza

S. Tommasi n.1, Coppito, 67100 L'Aquila, Italy
In mice with TNBS-induced intestinal fibrosis, tBHQ treatment downregulated TGF- $\beta 1$ expression, and phosphorylation of SMAD2/3 in a Nrf2-dependent manner, and significantly downregulated tissue inhibitor of metalloproteinases (TIMP)-1, but it did not affect matrix metalloproteinase (MMP).

Similar results were obtained in CCD-18Co fibroblasts stimulated with TGF- $\beta 1$. Moreover, Nrf 2 knockdown increased the expression of the TGF- $\beta 1 /$ Smad signaling pathway in CCD-18Co fibroblasts. These findings indicate that Nrf2 activation inhibited TGF- $\beta 1 /$ Smad signaling in intestinal fibrosis. In colon-derived CCD-18Co fibroblasts, Nrf2 attenuated intestinal fibrosis by inhibiting the ROSdependent TGF- $\beta 1$ signaling pathway via ROS scavenging. These in vitro results should also be confirmed using intestinal fibroblast cultures isolated from patients with fibrostenosing CD.

The Nrf2 inducer tBHQ has proven effective in preventing the development of intestinal fibrosis (prophylactic purpose). It would be crucial to ascertain whether it is also efficacious in treating established intestinal fibrosis in animal models (therapeutic purpose). To clarify whether the antifibrotic effect of Nrf2 induction is merely secondary to its anti-inflammatory action, and not a direct effect, it would be useful to compare its action on fibrogenesis in the acute and the chronic phase of intestinal inflammation induced by TNBS, during the first week and then at 6 and 12 weeks of treatment.

Furthermore, since Nrf2 is also involved in carcinogenesis in several organs, including the large bowel [8], the effects of Nrf2 activation observed in TNBS-induced colitis should also be replicated in dextran sodium sulfate-induced chronic colitis, which is also susceptible to evolve to CRC.

Taken as a whole, the above data suggest that Nrf2 could be a new target for the treatment of intestinal fibrosis.

\section{Compliance with ethical standards}

Conflict of interest The author has no conflicts of interest to declare and did not use any outside assistance in preparing the manuscript. 


\section{References}

1. Latella G, Di Gregorio J, Flati V, Rieder F, Lawrance IC. Mechanisms of initiation and progression of intestinal fibrosis in IBD. Scand J Gastroenterol. 2015;50:53-65.

2. Latella G, Rogler G, Bamias G, et al. Results of the 4th scientific workshop of the ECCO (I): pathophysiology of intestinal fibrosis in IBD. J Crohns Colitis. 2014;8:1147-1165.

3. Richter K, Kietzmann T. Reactive oxygen species and fibrosis: further evidence of a significant liaison. Cell Tissue Res. 2016;365:591-605.

4. Swamy SM, Rajasekaran NS, Thannickal VJ. Nuclear factorerythroid-2-related factor 2 in aging and lung fibrosis. Am J Pathol. 2016;186:1712-1723.
5. Liu RM, Desai LP. Reciprocal regulation of TGF- $\beta$ and reactive oxygen species: a perverse cycle for fibrosis. Redox Biol. 2015;6:565-577.

6. Ahamed J, Laurence J. Role of platelet-derived transforming growth factor- $\beta 1$ and reactive oxygen species in radiation-induced organ fibrosis. Antioxid Redox Signal. 2017;27:977-988.

7. Guan Y, Tan Y, Liu W, et al. NF-E2-related factor 2 suppresses intestinal fibrosis by inhibiting reactive oxygen species-dependent TGF- $\beta 1 /$ SMADs pathway. Dig Dis Sci. (Epub ahead of print). https://doi.org/10.1007/s10620-017-4710-z.

8. Sadeghi MR, Jeddi F, Soozangar N, Somi MH, Samadi N. The role of Nrf2-Keap1 axis in colorectal cancer, progression, and chemoresistance. Tumour Biol. 2017;39:1010428317705510. https://doi.org/10.1177/1010428317705510. 